\title{
FORTALECIENDO DESTREZAS LECTO ESCRITORAS A TRAVÉS DE ESTRATEGIAS PSICOPEDAGÓGICAS EN INFANTES DE 6 AÑOS
}

\section{STRENGTHENING LITERACY SKILLS - WRITERS THROUGH PSYCHO-PEDAGOGICAL STRATEGIES IN 6-YEAR-OLDS}

\section{FORTALECIMENTO DAS HABILIDADES LETIVAS - ESCRITORAS ATRAVÉS DE ESTRATÉGIAS PSICOPEDAGÓGICAS EM CRIANÇAS DE 6 ANOS}

Encuentre este artículo en:

http://revistas.uniminuto.edu/index.php/IYD

Para citar este artículo / To cite this article

Cruz, J. (2021). Fortaleciendo destrezas lecto escritoras a través de estrategias psicopedagógicas en infantes de 6 años. Inclusión \& Desarrollo, 8 (1), pp 83-94
Jenny Katherine Cruz Márquez katemarcruz@gmail.com Corporación Universitaria Minuto de Dios-UNIMINUTO

Bogotá- Colombia

Diagramación Sindy Catherine Charcas Ibarra

Fotografia portada Santiago Ardila Acero

\section{RESUMEN}

El presente artículo evidencia diferentes estrategias aplicadas mediante un proyecto de intervención de expertos en el aprendizaje lectoescritor a través de estrategias psicopedagógicas, para fortalecer falencias de dos estudiantes de seis años del Barrio Ciudad Roma del Liceo Infantil Sombrillitas De Colores, evidenciadas gracias a la utilización de test, entrevistas, análisis documentales y observación, las cuales se relacionaron con una metodología exploratoria no experimental, accediendo de manera natural por medio de la observación sin alterar las variables expuestas por las mismas. Los resultados reflejan avances significativos en el uso de herramientas lectoescritoras; sin embargo, todavía se debe generar aprendizaje constante para lograr una formación plena en cada una de las falencias manifestadas.

Palabras claves: intervención, lectoescritura, educación inicial, estrategias psicopedagógicas, rendimiento académico.

Fecha de recepción: 25 de septiembre de 2020 Fecha de aceptación: 9 de noviembre de 2020 Fecha de publicación: 1 de enero de 2021 


\section{ABSTRACT}

This article shows different strategies applied through a project of intervention of experts in the literacy learning through psychopedagogical strategies, to strengthen the failings of two six-year-old students from the Roma City District of the Liceo Infantil Sombrillitas De Colores, evidenced by the use of tests, interviews, documentary analysis and observation, which were related to a non-experimental exploratory methodology, accessing naturally by means of observation without altering the variables exposed by them. The results reflect significant advances in the use of reading and writing tools; however, constant learning must still be generated to achieve a full formation in each of the manifested shortcomings.

Keywords: intervention, literacy, initial education, psychopedagogical strategies, academic performance.

\section{SUMÀRIO}

O presente artigo evidencia diferentes estratégias aplicadas mediante um projeto de intervenção de especialistas no aprendizado lectoescritor através de estratégias psicopedagógicas, para fortalecer falências de dois estudantes de seis anos do Bairro Cidade Roma do Liceu Infantil Sombrinhas De Cores, evidenciadas graças à utilização de testes, entrevistas, análises documentais e observação, as quais se relacionaram com uma metodologia exploratória não experimental, acessando naturalmente por meio da observação sem alterar as variáveis expostas pelas mesmas. Os resultados refletem avanços significativos no uso de ferramentas lectoescritoras; no entanto, ainda é preciso gerar aprendizagem constante para conseguir uma formação plena em cada uma das falências manifestadas.

Palavras chave: intervenção, alfabetização, educação inicial, estratégias psicopedagógicas, desempenho acadêmico. 


\section{Introducción}

La lectoescritura es un paso esencial que permite el desarrollo del lenguaje de los niños, proporcionando una base exploratoria, en su entorno escolar y personal, es por eso que se busca adquirir esas estrategias psicopedagógicas que permitan mitigar e intervenir en el proceso de adquisición lecto escritor en niños de 6 años, puesto que es una etapa fundamental donde se evidencia con mayor exactitud en los infantes las dificultades que presentan al momento de iniciar a escribir o leer.

Para poder trabajar en las en las dificultades lectoescritoras en los niños es bueno evidenciar las razones de por qué un niño lleva alteraciones en su proceso, aunque debemos tener en cuenta que cada niño tiene su propio nivel y tiempo de aprendizaje, sin dejar a un lado que la mayoría de veces su dificultad lecto escritora es ocasionado por múltiples alteraciones de lateralidad, percepción ,psicomotricidad,atención,memoria entre otras, a lo que se recomienda iniciar un refuerzo en cada una de esas áreas mencionadas anteriormente; desde las primeras etapas de desarrollo de los infantes. Es así como surge la iniciativa de buscar alternativas para trabajar frente a la recopilación de diferentes estrategias aplicadas a lo largo de la psicopedagogía, para fortalecer las diferentes falencias observadas e identificadas a dos niños de 6 años mediante de la intervención a realizar, y lograr como docente en formación estrategias para implementar diferentes acciones de mejora frente a las problemáticas evidenciadas de acuerdo a la observación de sus dificultades lectoescritoras reflejadas en la mediación de la misma.

\section{Referentes conceptuales}

Cuando se dice que un niño no lee ni escribe bien, es una afirmación que puede referirse a una infinidad de cosas que no corresponde a la problemática, aislando la posibilidad de encontrar estrategias para intervenir en la contrariedad presentada, debido a que sus dificultades son tratadas desde un punto de vista neurológico, lo que generalmente plantea un retraso por parte del infante. Lo anteriormente mencionado, se asemeja a épocas remotas por diferentes psicólogos y pedágogos como Hinshel Wood y Morgan en 1896. En la actualidad a través de diferentes investigaciones realizadas se tiene en cuenta el ritmo de maduración y desarrollo del infante, en donde la intervención es enfocada al aprestamiento, desde la educación inicial, mediante ayuda de múltiples ejercicios preparatorios antes de iniciar a escribir o a leer por parte de los niños, realmente ahí, es donde se ha presentado la mayoría de fallas, debido a padres y maestros apresurados en dar este paso sin armonizar esas propuestas pedagógicas y psicológicas en las actividades cotidianas del niño, que permiten que interioricen en sus habilidades motrices o de lenguaje obteniendo en un futuro el dominio del mismo con facilidad.

Al momento de plantear una intervención se evidencia varios modelos teóricos para la mitigación de las dificultades lectoescritoras en niños de 6 años. Refiriendo a diferentes métodos que trabajan la estimulación, a través de la motivación, siendo de gran ayuda en diferentes instituciones de América Latina e inclusive puesta en práctica por algunos docentes colombianos, donde las figuras y formas son inherentes a la percepción, coordinación y constancia perceptual visual ,centrándose en el aprendizaje de los niños.

Emilia Ferreiro (2001) hace su aporte a través de diferentes fases para la construcción lectoescritora, haciendo una intervención profunda en fortalecer falencias a partir de una propuesta de intervención centrada en la lúdica 
por medio estrategias psicopedagógicas, así lo demostró en un grado preescolar en niños no escolarizados de aproximadamente de 5 a 6 años de edad, en donde puso a practicar los diferentes niveles de lectura y escritura influenciando a través del psicogénesis de escritura e imágenes a los niños, para que generarán grafismo evidenciando resultados formativos importantes para los mismos. A lo que el pedágogo Joaquín Montano (2013, p.19) afirma:

Para trabajar en el proceso lecto escritor de Emilia Ferreiro en los niños, se debe enfocar en la lengua escrita a través de las diferentes recomendaciones pedagógicas como la psicogénesis del sistema de escritura. El cual se relaciona con un método que también ayuda en este proceso de una manera novedosa de aprender, como lo es el método que propone el reconocido Juan Carlos Negret, el cual ha sido aplicado a 137 centros educativos, siendo un método que promete motivación para los niños iniciando el proceso con el garabateo hasta la introducción de las consonantes, el cual es llamado el programa de letras, teniendo como objetivo entender los conocimientos propios sobre la lectura y escritura de los niños. A lo largo de los años, uno de los resultados relevantes de sus intervenciones se le alude al trabajo que realizaron estudiantes de la Universidad Nacional De Colombia, en su maestría de educación de Ciencias Humanas, a niños de grado de primero en donde plasma lo efectivo que fue trabajar con el programa desde una perspectiva constructivista y didáctica que manifiestan Diana Calderón y María Torres (2015, p.20) en el siguiente apartado:

La utilización de los formatos gráficos de los textos, el uso de los renglones, el aprendizaje de la forma, tipo, caso y tamaño de las letras convencionales que se usan en la escritura alfabética, y por último, el aprendizaje de los códigos escriturales silábico y alfabético de Juan Carlos Negret permitió la efectividad para moderar las diferentes dificultades lecto escritoras, evidenciadas en niños de 6 años del grado primero Jardín Mundo Mágico A, B, C.

El escritor Rubén Darío Hurtado Vergara, un pedágogo colombiano ,que trabaja algo similar a lo que mencionamos anteriormente del método Negret, invita a trabajar desde enfoques de comunicación y expresión desde la educación inicial como una alternativa constructivista donde estimula el diálogo con los niños de acuerdo a sus experiencias y creatividad permitiendo un eje para intervenir en las dificultades lecto escritoras el cual lo afirma Hurtado (2016, p.23) :

Las experiencias de los niños son posibilidades que a través de la escucha y expresión permiten ejercer estrategias factibles en su adquisición de lenguaje y escritura ya que es una premisa para diseñar material didáctico y llamativo frente a las diferentes dificultades de los niños.

A lo largo de los años se ha desarrollado múltiples métodos y herramientas para la dificultad lecto escritora, aprobando un sentido lineal al momento de implementar diferentes estrategias en donde los niños trabajan en factores que conlleva a la misma, muchas de ellas tal vez no son de autores reconocidos, pero son eficaces al momento de influir en los procesos de adquisición lecto escritora en los niños, como el de la Psicopedagoga Victoria Bermúdez de la Institución Educativa Manuel Cepeda Vargas de Bogotá D.C, la cual creó una cartilla en donde expone diferentes actividades psicopedagogas, llamativas para que los estudiantes con una alguna dificultad lectoescritora puedan apropiarse de ella y les permita fomentar su aprendizaje en compañía de sus padres a lo que ha obtenido resultados fructíferos en varios niños, los cuales han 
logrado mejorar su rendimiento académico.

De acuerdo a cada uno de los referentes, podemos deducir que es algo realmente necesario a todas las teorías o métodos mencionados sirven para participar activamente en el proceso de intervención de los niños de 6 años, siendo ejes que son altamente calificables, apoyando diferentes perspectivas psicopedagógicas, proporcionado la lectura y escritura de manera correcta en las primeras etapas iniciales de los niños, ejerciendo desarrollo y oportunidades para los infantes que actualmente evidencian diferentes dificultades, generando nuevos conceptos del por qué se presenta este impedimento en la actualidad para trabajar en las diferentes problemáticas lectoescritoras generadas por la misma.

Además, que la vinculación de docentes, psicólogos y padres en este proceso es de suma importancia para generar alternativas para mitigar cada una de esas falencias a lo que es importante implementar lo que afirma de una forma precisa y gráfica la escritora norteamericana Buchwald ( 2010, p.1 ),“Los niños se hacen lectores en el regazo de sus padres."

\section{Metodología}

El método de la presente propuesta de intervención está enfocado a una evaluación de una intervención pre, post ,a través de la recopilación de estrategias psicopedagógicas planteadas por diferentes metodologías aplicadas por psicólogos y pedágogos en niños de 6 años, mitigando diferentes dificultades de aprendizaje lectoescritor,generando variedad de actividades ejecutadas a través de cinco sesiones, durante un mes, con una intervención semanal, con duración de una hora, permitiendo trabajar áreas como la percepción visual, desarrollo motor y cognitivo.

\section{Participantes}

La intervención se realizó a través de la población seleccionada de dos estudiantes del grado primero de 6 años del Liceo Infantil Sombrillitas De Colores del Barrio Ciudad Roma, cuyos nombres son Santiago y Sergio Rey Trujillo los cuales evidencian diferentes dificultades lectoescritoras, junto a sus dos padres de familia Carlos Andrés Rey y Paola Andrea Trujillo los cuales incentivaron a sus hijos a participar de manera activa en las diferentes actividades presentadas durante el desarrollo de la propuesta de intervención.

\section{Instrumentos}

En busca de mayor veracidad de información, antes de iniciar la intervención, se implementó una recolección de datos mixta, a través de diferentes herramientas, como: una entrevista estructurada a padres de familia, para crear una concepción de los diferentes comportamientos en su rendimiento escolar. La observación directa y análisis documental que permitieron analizar todo el ambiente en donde inició las dificultades de los infantes, contribuyendo a estudiar qué estrategias eran las más prudentes para la intervención propuesta, generando información relevante sobre el proceso lectoescritor de los niños a través de la identificación de aspectos iniciales de las problemáticas a lo largo de su adquisición lecto escritora.

Sin embargo, es bueno resaltar que los instrumentos más beneficiosos al iniciar fueron los pre test, como el de Lorenzo Filbo que generó un gran aporte para patentizar que contrariedades manifestaban los niños en su proceso lecto escritor gracias a su formulación, la cual está destinada a medir a juicio del autor, la madurez de un niño para enfrentar la lectoescritura. Intentando predecir el tiempo que tardará el niño en adquirir ambos aprendizajes. 
El test concede un máximo de 24 puntos, el cual obtuvo en los dos infantes un puntaje en términos medio en donde la prueba de Mabel Condemarin corroborar estos resultados a través de su prueba visual y motora, de dos hojas que consistía en escribir, recortar, describir y tomar dictado la cual demostró que los infantes evidenciaban dificultades motoras, visuales y de coordinación en pautas lectoras.

Durante la intervención se generaron estrategias psicopedagógicas fundamentadas en varios pedágogos y psicólogos como Juan Carlos Negret, Emilia Ferreiro, Rubén Hurtado, que a lo largo de los años con sus actividades han generado grandes soluciones para dificultades lecto escritoras en niños en educación inicial, las cuales a través de cinco sesiones, en el transcurso del mes de Julio, se aplicaron mediante un lapso de una hora, divididas en tres etapas fundamentales que fueron estimulación lecto escritora, didáctica e integración a través de ejercicios psicopedagógicos a padres de familias con los infantes. Para culminar se dio lugar a la evaluación final para evaluar la efectividad de las diferentes actividades psicopedagógicas ejecutadas en las diferentes sesiones, la cual permitió identificar el avance e impacto que generó las actividades durante la ejecución de la intervención.
Figura.1
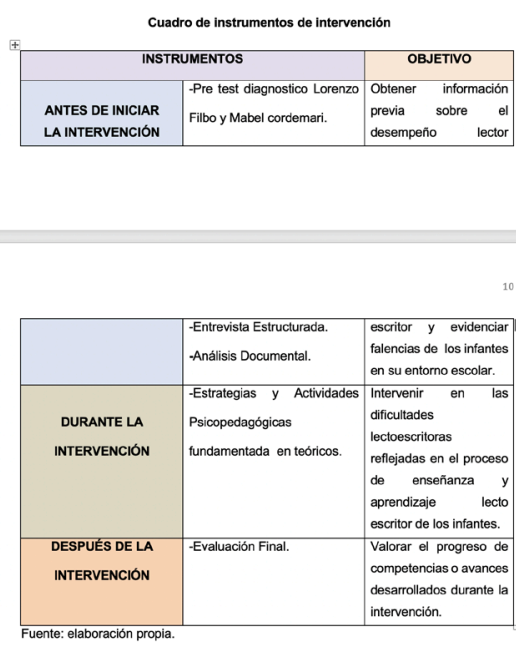

\section{Diseño de la propuesta de intervención}

Existen múltiples enfoques teóricos para intervenir en las dificultades lectoescritoras en los niños, sin embargo, las estrategias psicopedagógicas fundamentadas en varios teóricos permite fortalecer múltiples áreas, en donde los infantes de seis años evidencian mayor dificultad en su aprendizaje como la percepción visual, coordinación, percepción espacial y motricidad, permitiendo una interacción constante con padres de familia que incentiva a generar progresos en cada una de falencias identificadas e ideas para seguir fomentando en casa el proceso lecto escritor de manera lúdica y didáctica.

La propuesta de intervención está dividida en tres fases divididas en cinco sesiones, complementadas con 10 actividades psicopedagógicas evidenciadas en la figura 2, que sirven para trabajar diferentes arias que generan dificultad lecto escritora, plasmadas en cinco sesiones, durante un mes, produciendo avances significativos en la dificultades lectoescritoras en niños de 6 años, identificadas en los pretest e información recopilada al iniciar la intervención. 


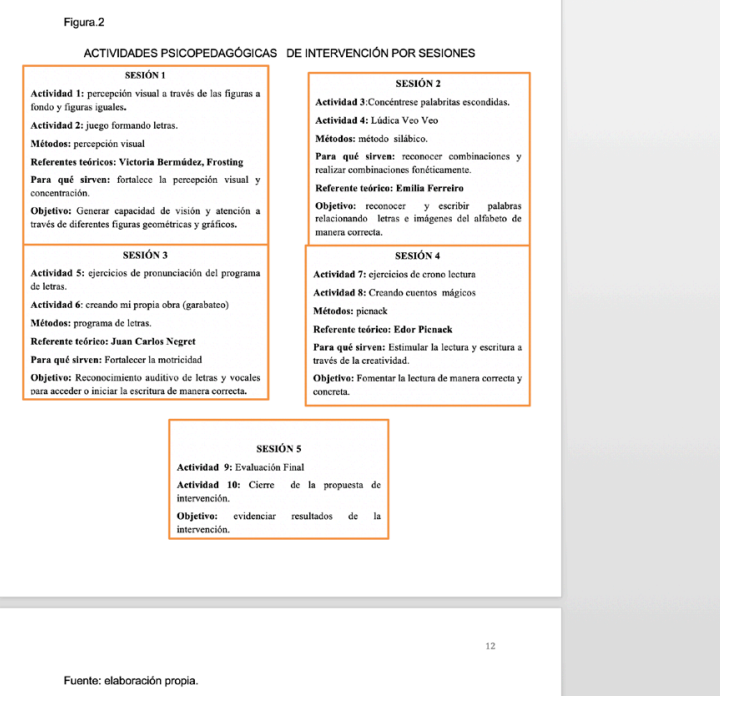

Procedimiento de la propuesta de intervención

Primera fase: se realizó una presentación formal como docente en formación de la Corporación Universitaria Minuto De Dios Uniminuto de último de semestre de pedagogía infantil, a padres y niños por medio de una interacción virtual en la plataforma Zoom donde se realizó una entrevista de aproximadamente de 10 preguntas a padres de familia e infantes con el objetivo de obtener inquisición de características previas al entorno. Además de manifestar de manera diligente la presentación de la propuesta de intervención, lectoescritora a través de un cronograma evidenciando las sesiones y duración de las diferentes actividades propuestas.

Para finalizar, esta primera fase se realizó dos pres test de Lorenzo Filbo y Mabel Condemarín, muy conocidos alrededor de Latino América, en donde se exhibió un protocolo a través de diferentes actividades de recorte, lectura estandarizada y grafismo, en donde los niños reflejaron que dificultades de aprendizaje lecto escritoras están evidenciando actualmente y cuál de todas las tareas realizadas fueron sus favoritas.
Segunda fase: retomando las acciones realizadas en la primera fase el 3 de julio del 2020 se da inicio a las 5 sesiones, dando lugar a las estrategias psicopedagógicas lectoescritoras para iniciar la intervención de acuerdo a las falencias y resultados evidenciados en los Pre Test.

Sesión 1: en la presente sesión se inició con una dinámica rompe hielo saludo del oso en donde se pretendió que los niños generaran atención y atracción a las actividades propuestas abriendo campo para el desarrollo de la actividad número uno de percepción visual a través de fondos y figuras de Frosting ,la cual pretende generar capacidad de atención, estabilizar la visión en los niños, teniendo en cuenta que muchos niños se les dificulta generar percepción en sus primeros años escolares obstaculizando sus procesos de aprendizaje. Se utilizaron recursos como lápiz, colores y una guía en donde evidenciaba las diferentes formas geométricas en donde los niños procedieron a desarrollarla en compañía de los padres de familia de acuerdo a las instrucciones dadas durante la sesión retroalimentando la importancia de la vista y los resultados de la actividad de acuerdo a la experiencia propia y del referente teórico, finalizando con el juego formado letras en donde se incentivó a los padres a seguir manejando este tipo de juegos porque permite producir fluidez, atención y comprensión de sílabas a través de canciones o memofichas en donde los niños por medio de la concentración de acuerdo a frases y sílabas, emitidas por rondas infantiles, combinando los diferentes fonemas permitiéndoles construir palabras, un gran aporte que generó la psicopedagoga Victoria Bermudez la cual obtenido resultados fructuosos en sus estudiantes de primero y preescolar, en su intervención escolar frente al proceso lecto escritor en el Colegio Manuel Cepeda Vargas. 
Sesión 2: se realizó la continuación de ejercicios silábicos a través de actividades expuestas por Emilia Ferreiro con el objetivo de fortalecer la lectura y escritura mediante actividades lúdicas como un concéntrese de palabras e imágenes que están divididos por sílabas, en donde los niños al encontrar las imágenes semejantes, complementará diferentes palabras a través de la unión de sílabas, una actividad que ha causado furor en muchas instituciones por lo llamativa y que permite resultados positivos para el aprendizaje de los infantes; para culminar se desarrolló el Juego Veo Veo el cual permite interactuar con las letras del alfabeto de manera auditiva a través de diferentes ritmos de canciones, en donde las menciona y a través de un tiempo de cinco minutos los niños escribirán palabras por las primeras letras que escuchen, en donde la madre de los infantes se integró sugiriendo ideas para una próxima actividad además de compartir su experiencia de cómo sirvió la actividad para mitigar las falencias de sus hijos en donde reiteró que las tomara como apoyo para seguir contribuyendo en el proceso.

En las siguientes sesiones se realizaron ejercicios de pronunciación, expresión, manejo de renglón y cronolectura de acuerdo a falencias identificadas y expresadas por los padres de familia en el transcurso de las dos sesiones anteriores.

Sesión 3: en esta sesión se trabajó una de las actividades realizadas por Juan Carlos Negret, en el programa de letras en la cual hace alusión al método Alfa, en donde los participantes por medio de una lotería didáctica pronuncian en voz alta las diferentes letras del abecedario y frases encontradas, alternándose por turnos, a lo que los niños reaccionaron con gran entusiasmo expresado a través de un pequeño escrito y dibujo de garabateo libre para hacer cierre de la sesión se generó una par de recomendaciones a los padres, haciendo énfasis de la importancia de estimular a los niños en las primeras etapas de vida con ejercicios de motricidad y vocabulario creativas con el objetivo de mitigar dificultades lectoescritoras, finalizando con un detalle para Sergio y Santiago de un libro de colorear para seguir incentivando a su aprendizaje.

Sesión 4 :Para dar comienzo al penúltimo encuentro se pudo contar con un poco más de tiempo para hacer un repaso de algunas actividades realizadas anteriormente, además de iniciar de una manera poco diferente en dónde el encargado de la actividades del día fue un títere llamado Lecto, en donde explico a los niños como pronunciar y escribir de manera correcta, apoyándose en actividades de crono lectura inventada por el pedágogo Edor Pinack, la cual consiste en cronometrar un minuto de lectura contando el número de palabras leídas, repitiendo el mismo proceso mediante un cuento creado por ellos en donde su objetivo es aumentar la comprensión de palabras a través del uso del renglón y la creatividad.

Sesión 5: en la última sesión, se genera la evaluación final por medio de la realización de una guía que involucra los temas vistos en las anteriores sesiones, con el fin de analizar cuál fue el avance que obtuvieron los niños durante el desarrollo de la intervención, luego se procedió a realizar un juego interactivo de lecto escritura, el cual generó una mención para los niños por su compromiso de aprender, dando paso a la culminación de la intervención a través de recomendaciones finales para padres e infantes de acuerdo a las reacciones, procesos y falencias durante la aplicación de las actividades propuestas en dónde se incentivó a seguir con el proceso mediante la adquisición de talleres de apoyo que permita avanzar en el aprendizaje de los niños y gerando conciencia de la importancia del acompañamiento de padres de familia en el proceso de enseñanza para los hijos mediante una reflexión de Pipo 
Hernández, llamada "Hablé Con Su Hijo", dando lugar para cerrar la ejecución de la propuesta de intervención con agradecimientos y sugerencias para una próxima oportunidad de acuerdo aspectos negativos y positivos vividos durante la experiencia de la ejecución de las diferentes actividades.

\section{Resultados}

A continuación se evidencia cada uno de los resultados de acuerdo a instrumentos y actividades ejecutadas de inicio a final en la ejecución de la propuesta de intervención.

Resultado entrevista a padres: mediante la entrevista pudimos identificar algunos aspectos importantes de los niños como fue su origen ,cual es relación que mantienen como hermanos, además de conocer que tipos comportamientos ha presentado en su entorno escolar que ha permitido a docentes y padres expresar diferentes falencias en su ambiente lecto escritor, en donde la madre nos manifestaba que Sergio y Santiago se les dificulta el uso de renglón, leer de manera correcta además de realizar combinaciones de palabras lo que ha afectado su rendimiento académico.

Resultado entrevista a infantes: a través de cortas preguntas los infantes expresaron porque se les dificulta leer y escribir lo cual uno de los niños manifestó que veía borroso y el otro que no escribía de manera correcta por que las actividades que la docente planteaba en su colegio era muy estrictas y poco llamativas lo que generaba pereza en aprender.

Resultado pretest de Lorenzo Filbo y Mabel Condermari: se aplicaron pruebas de dictado, recorte y lectura según los solicitado en el desarrollo de los pretest el cual arrojo un puntaje de nivel medio, en donde se reflejó que el infante Santiago Rey presenta dificultades de percepción visual con mayor elevación a diferencia de su hermano Sergio sin embargo en los dos infantes se evidencio dificultades de motricidad en un alto grado los cuales dificulta el uso de renglón, coordinación y de percepción espacial.

Resultados en la ejecución de actividades psicopedagógicas de la propuesta de intervención: A continuación, se evidenciaran resultados y logros obtenidos mediante la aplicación de la propuesta durante las cinco sesiones, programadas de una hora, durante un mes en un lapso de una vez a la semana.

Sesión 1: al momento de realizar los ejercicios de percepción visual se obtuvo atención de los infantes generando interés por aprender, además permitió generar avances muy leves frente a la percepción visual, lo que incentivo a padres de familia seguir reforzando en casa con diferentes actividades similares a la planteada en la sesión.

Sesión 2: a través de la actividad propuesta silábica de Emilia Ferreiro y la lúdica Veo Veo, en los infantes se fortaleció la lectoescritura obteniendo como resultado cierta empatía por parte de los infantes, sin embargo a raíz de sus falencias se estableció ejercicios en casa como refuerzo para su revisión dentro de la próxima sesión.

Sesión 3: en la sesión se revisó las tareas de refuerzo de la propuestas en el encuentro anterior, las cuales junto a la actividad desarrollada del programa de letras de Juan Carlos Negret, se obtuvo una connotación positiva permitiendo arrojar un resultado en donde los infantes dejan ver como a través de las actividades ejecutadas logran reconocer las letras de abecedario de manera correcta, además de escribir cortas, pero correctas palabras apoyado en la actividad de garabateo libre. 
Sesión 4: durante el desarrollo de las ante penúltimas actividades los niños evidenciaron un leve avance que causó gran satisfacción en los padres de familia, que se reflejó en la actividad de cronolectura, en donde los niños leyeron y escribieron de manera correcta algunas palabras, sin embargo se reiteró a padres de familia que toca seguir trabajando con los infantes combinación de palabras para lograr mitigar las falencias en su totalidad.

Sesión 5: en la última sesión se obtuvo resultados positivos gracias algunos avances que evidenciaron los niños como reconocer y leer algunas palabras, escribir de manera ordenada intermitententemente en el renglón, algo que anteriormente se les dificultaba un poco más, pero que con constancia y apoyo de los padres lo lograrán en su totalidad, además de obtener el agradecimiento de los padres de familia los cuales se comprometieron en seguir las recomendaciones propuestas durante el desarrollo de la intervención para disminuir las problemáticas presentadas, para mejorar el proceso lecto escritor de Santiago y Sergio.

\section{Resultados evaluación final de la} intervención: la evaluación evidenció logros leves, pero significativos, en donde refleja como los infantes comprendieron los temas expuestos a lo largo de intervención mediante la prueba compuesta por 10 puntos la cual solicitaba varios ejercicios de percepción visual,espacial,silábico, coordinación y motricidad de desarrollo individual y autónomo por los infantes, generando como resultado un puntaje de seis sobre 10 en Sergio y 5 sobre 10 en Santiago en donde se recomienda que los niños deben todavía fortalecer aspectos motrices y de percepción visual, sin embargo obtuvieron connotación positiva en los puntos silábicos, percepción espacial y de coordinación lo que se incentivó a padres a seguir trabajando con cada uno de los niños los aspectos los cuales resultaron bajos y seguir vigorizando los positivos.

\section{Discusión}

Los resultados obtenidos en la intervención a lo largo decada fase y sesión ejecutada, evidencian que cada una de las actividades permite generar avances leves en los infantes frente a las dificultades presentadas por los mismos, pero que si se aplicaran con constancia podrían mitigar las falencias por completo. Además nos ayuda a comprobar que la metodología de usar varios métodos propuestos por pedagogos y psicólogos nos apoya a trabajar varios enfoques como la estimulación a través de la motivación, siendo de gran ayuda en diferentes instituciones de América Latina e inclusive puesta en práctica por algunos docentes colombianos, y en esta ocasión en la propuesta de intervención ejecutada, en donde los métodos de Emilia Ferreiro, Juan Carlos Negret, Victoria Bermúdez ,Frosting y centrándose en el aprendizaje de los niños a través de la dificultades evidenciadas en los Pres Test de Mabel Cordemari , Lorenzo Filbo y en problemas comunes en niños de 6 años al iniciar su proceso lecto escritor, generan un resultado de aprendizaje llamativo e interesante en los niños a través de actividades lúdicas y didácticas que permiten que los padres también interactúe en el proceso de mejora de sus hijos, originando una enseñanza y aprendizaje significativa en los infantes.

También es importante resaltar que la oportunidad que se presenta durante el proceso de la intervención de conocer el origen de las dificultades de los niños porque generan ideas de cómo preparar las sesiones de tal manera que todos los participantes se involucren, estableciendo un ambiente de aprendizaje más apropiado en los infantes, causando confianza pero sobre todo captar el interés dándoles a conocer de lo fructuosa que puede resultar la aplicación de las actividades de intervención si se trabaja de manera grupal, en donde todos participen desde sus gustos, necesidades lecto 
escritoras pero sobre todo desde su opinión crítica constructiva.

\section{Conclusiones}

- La presente propuesta de intervención de estrategias psicopedagógicas permitió trabajar en diferentes problemáticas comunes en niños de seis en años en la iniciación del aprendizaje lectoescritor como lo es la motricidad, coordinación, percepción visual y espacial, generando diferentes métodos psicopedagógicos lectoescritores enfocando diferentes áreas que dificultan el aprendizaje en los niños.

- Durante la ejecución de las actividades propuestas se reflejó una participación por parte de los infantes y padres de familia.

- Las actividades lúdicas y didácticas contribuyen a la mitigación de dificultades de aprendizaje de acuerdo con que genera gran interés por parte de los infantes generando avances significativos frente a las falencias identificadas en los niños a través de las sesiones y desarrollo de las actividades propuestas.

- Se percibiólaimportancia delacompañamiento de padres a familia para la intervención, los cuales ayudan incentivar a sus hijos en fortalecer cada una de las problemáticas que presentan.

- Hay satisfacción por parte de los padres e infantes de la manera que se desarrolló la propuesta de intervención. 


\section{Referencias bibliográficas}

Álvarez, Á., \& Orellano, E. (1979). Desarrollo de las funciones básicas para el aprendizaje de la lectoescritura según la teoría de Piaget. Segunda parte. Revista Latinoamericana de Psicología, 11(2), 249-259.

Gutiérrez Melgarejo, D. I., \& Neuta García, K. A. (2015). Prevalencia de las habilidades perceptuales visuales, la integración viso-motora, los movimientos sacádicos, la atención visual y el proceso de lecto-escritura en niños entre 6-7 años de la ciudad de Bogotá en estratos 5 y 6.

Abril Bocachica, J., \& Sanabria Pinzón, G. P. (2018). Construcción del proceso lecto escritor en niños de primer grado del colegio Agustiniana Norte a través del programa letras.

Ferreiro, E., \& Teberosky, A. (1991). Los sistemas de escritura en el desarrollo del niño. Siglo XXI.

Colmenares E, A. M. (2012). Investigación-acción participativa: una metodología integradora del conocimiento y la acción. Voces y Silencios. Revista Latinoamericana de Educación, 3(1), 102- 115.

Hurtado Vergara, R. D. (2005). El recuento, el resumen y las preguntas: estrategias didácticas para mejorar la comprensión de lectura. Lenguaje. Emilia Buchwald. (2010).El papel de los padres de lectura.: Universidad Amaranta:Recuperadode:https://www.uv.mx/epl/files/2015/08/TRABAJO_RECEPCIONAL_AMARANTA.pdf 